\title{
The Scholarly Commons - principles and practices to guide research communication
}

(iD Jeroen Bosman, iD Ian Bruno, iD Chris Chapman, iD Bastian Greshake Tzovaras, iD Nate Jacobs, (D Bianca Kramer, iD Maryann Martone, iD Fiona Murphy, iD Daniel Paul O'Donnell, iD Michael Bar-Sinai, iD Stephanie Hagstrom, iD Josh Utley, Lusia Veksler

Authorship contributor roles (alphabetically, using the CRediT contributor roles taxonomy and visualizations thereof from Mozilla Science contributor badges)

Conceptualization - Bosman, Bruno, Chapman, Greshake Tzovaras, Hagstrom, Jacobs, Kramer, Martone, Murphy, O'Donnell

Funding acquisition - Hagstrom, Martone

Project administration - Hagstrom, Veksler

Resources - Bar-Sinai, Bosman, Bruno, Chapman, Greshake Tzovaras, Hagstrom, Jacobs, Kramer, Martone, Murphy, O'Donnell, Utley, Veksler

Software - Bar-Sinai, Utley

Supervision - Bosman, Bruno, Chapman, Greshake Tzovaras, Hagstrom, Jacobs, Kramer, Martone, Murphy, O'Donnell;

Visualization - Bar-Sinai, Bosman, Kramer, Utley

Writing - original draft - Bosman, Chapman, Kramer, Martone, Murphy; O'Donnell

Writing - review \& editing - Bosman, Bruno, Chapman, Greshake Tzovaras, Jacobs, Kramer, Martone, Murphy, O'Donnell

\footnotetext{
Author affiliations

Bosman - Utrecht University Library, Utrecht, The Netherlands j.bosman@uu.nl Bruno - Cambridge Crystallographic Data Centre, Cambridge, United Kingdom Chapman - Pentandra Research Solutions, Logan UT, United States Greshake Tzovaras - Goethe-Universität Frankfurt am Main, Frankfurt am Main, Germany Jacobs - University of California, Irvine, United States Kramer - Utrecht University Library, Utrecht, The Netherlands b.m.r.kramer@uu.nl Martone - University of California San Diego, La Jolla CA, United States Murphy - University of Reading, Reading, United Kingdom
} 
O'Donnell - University of Lethbridge, Lethbridge AB, Canada

Bar-Sinai - Ben-Gurion University of the Negev, Be'er-Sheva, Israel

Hagstrom - University of California San Diego, La Jolla CA, United States

Utley - Intrepid Network, San Diego CA, United States.

Veksler - University of California San Diego, La Jolla CA, United States

\section{Conflicts of interest}

Bosman, Bruno, Chapman, Greshake Tzovaras, Jacobs, Kramer, Martone, Murphy, and O'Donnell are all members of the steering committee of the FORCE11 Scholarly

Communication Working Group. Bar-Sinai is developing the current decision tree software for FORCE11, and a member of the Data Science Team at the Institute for Quantitative Social Science (IQSS) at Harvard University involving developing the Dataverse data repository software. Bosman is a subject editor for the RIO Journal. Chapman is a co-founder of Pentandra, a social business seeking to open up the knowledge of the world. Martone is director of Biosciences at Hypothesis, a non-profit developing technology to annotate the web, and a founder of SciCrunch.com, a technology company that provides technology for biomedical research resources. Murphy is board member of the Dryad Data Repository and project manager for data2paper which is developing a data publishing app.

\section{Keywords}

scholarly communication, research communication, scholarly commons, principles, decision trees, inclusivity

\section{Abstract}

Despite all available technology and despite major disruptions that the internet brought about in many sectors of modern life, scholarly communication has only seen change at glacial pace. Many useful, laudable tools and services are being developed to solve specific issues for particular domain groups. However, the question of how these efforts fit together remains largely unaddressed. If we have alternative models for all parts of the system, will that result in a coherent system? Will it be interoperable? Will it appeal to people as viable alternative? Will it be open and participatory for all?

The solution we propose is that of a scholarly commons: a set of principles and rules for the community of researchers and other stakeholders to ascribe to, the practices based on those principles, and the common pool of resources around which the principles and practices revolve. The tenets of the scholarly commons are that research and knowledge should be freely available to all who wish to use or reuse it (open, FAIR and citable), participation in the production and use of knowledge should be open to all who wish to participate, and there should be no systemic barriers and disincentives to prevent either such free use or open participation. 
In this paper, we outline the backgrounds of the idea of the scholarly commons and the various considerations that play a role in defining it. We share the principles of the scholarly commons and the degrees of freedom interpreting those principles, and consider the broader landscape of ideas and charters that the scholarly commons fits into. Finally, we present a call for action to involve like-minded people in the discussion on how to bring such a commons to fruition, and what this would mean for different communities within science and scholarship.

\section{Introduction: The need for a principled alternative}

This should be the Golden Age of Scholarly Communication. Information should be easier to access and more complete than even before in human history--a broadband enabled smart phone puts more information in the palm of your hand than was previously available in any of the greatest historical libraries - given access and absence of censorship.

The same is true of publication. When in the past, the dissemination of knowledge required costly raw material and services such as paper and post, digital technologies have reduced costs of dissemination (and in theory at least, production and publication as a whole), and many have imagined how these costs might be open to further disruption (for a variety of perspectives, see Research Information Network 2008; Conley \& Wooders 2009; Morris 2005; O’Donnell et al. 2015).

The origin of these changes is, of course, the development of the World Wide Web - a technology originally designed for document management at a government research lab that has subsequently disrupted in fundamental ways most aspects of commerce, travel, industry, media and communication. The original proposal for the worldwide web reads:

.... the hope would be to allow a pool of information to develop which could grow and evolve with the organisation and the projects it describes. For this to be possible, the method of storage must not place its own restraints on the information (Berners-Lee 1989).

This sounds remarkably like a good way to organize scholarly information e.g. as described by Hess \& Ostrom (Hess \& Ostrom 2007). But despite the available technology and despite major disruptions that the internet brought about in many sectors of modern life, scholarly communication has only seen change at glacial pace.

We first saw the serials crisis, then the funding/evaluation crisis and finally the reproducibility crisis. Open Access has been the answer to the first; 23 years of it brought us to a level of $45 \%$, but only for recent publications and only in the widest definitions of Open Access (Piwowar et al. 2017). The funding/evaluation crisis still lingers on, with impact factors and publish or perish motives driving researcher practices across the globe, despite laudable initiatives such as The San Francisco Declaration on Research Assessment. The reproducibility crisis is shaking up the core of science, whether it's psychology, cancer research or economics - data sharing and 
reproducible workflows are seeing a very slow introduction. The blame for this slow pace of change is often put on incentive structures, or even broader, the slow "cultural change". However, this does not dismiss us from the task to try to dissect what is going on exactly and overcome the stagnation, particularly as the incentive structure was developed and is maintained by scholars for scholars. Those outside the academy that have a stake in scholarly communication - e.g. the public and the governments that fund scholarship - arguably care about the best scholarship and access to it, and less about promotion and tenure processes or self-imposed rules and restrictions such as author position. The system is ultimately driven by scholars themselves.

Of course we can wait for these problems to get a mainstream solution, and indeed work should continue on that route, with funders and governments creating policies on open access and open data. However, we also see solutions being created in parallel to the reigning system, perhaps inspired by the saying attributed to Buckminster Fuller: "You never change things by fighting the existing reality. To change something, build a new model that makes the existing model obsolete". Over the last quarter century we have witnessed the introduction of many new models. We now have journals with new models (e.g. PeerJ, RIO, F1000 and more generally journals based on open source software as $\underline{\mathrm{OJS}}$ ), we see social networks for researchers springing up (e.g. ResearchGate, Humanities Commons), we see new systems for early paper sharing (first Arxiv, SSRN and RePEC, now BioRxiv and many more), we see discovery systems built on a collaborative model (e.g. PhilPapers), novel and open modes of peer review and

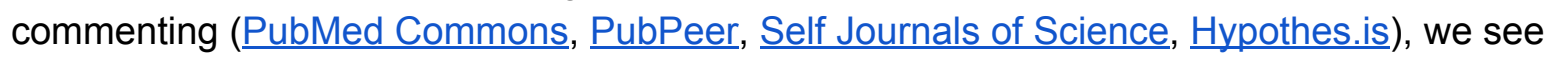
initiatives trying to change reward and evaluation systems (Publons, journals as Collabra, altmetrics providers such as Altmetric and ImpactStory), collaborative writing systems as Authorea, and hundreds more (Bosman \& Kramer 2015).

The good news is that some stakeholders in the mainstream system, funders such as Wellcome, governing institutions such as the European Union, have started to embrace and even drive some of these new ideas. However, this is much less the case with legacy publishers and learned societies - these tend only to embrace new ideas insofar as they do not change their core model (e.g. supporting $\underline{\mathrm{ORCID}}, \underline{\mathrm{DO}}$ and various $\underline{\mathrm{CC} \text {-licenses) }}$ or can be offered strategically as an option (open access or open or portable peer review).

Another problem with the myriad of new tools and platforms is that they solve one aspect of the system, from one (disciplinary or cultural) perspective at a time. Some make one aspect of scholarly communication more open, others add some efficiency, still others try to improve reproducibility, often geared at a specific field such as biomedical sciences or psychology. But the biggest problem is perhaps that it is not at all clear what kind of system these new models together will produce. If we have alternative models for all parts of the system, will that result in a coherent system? Will it be interoperable? Will it appeal to people as a viable alternative?

Finally, many new initiatives are still driven by business models and the current incentive structure of scholarship, and less by the need of the scholarly community itself. What do we as scholars want to do? How can we take advantage of modern technologies - which deliver 
tremendous communication and computation power into the hands of individuals - to make our scholarship better and to fulfill our mission as scholars?

The solution we propose is that of a scholarly commons: a set of principles and rules for the community of researchers and other stakeholders to ascribe to, the practices based on those principles, and the common pool of resources around which the principles and practices revolve.

This common pool of resources is to be freely used by anybody, but not to be extracted from by anybody (cf. (Ostrom 1990)), making it sustainable in the long term. In a digital context, subtractability of knowledge is low, meaning that it can be used by many without depleting the source. The scholarly commons also includes the notion that the organisation of resources should be such that the potential for collaboration, reusing and building on shared research objects is maximised. Consequently, it not only aims at making sure that research objects are freely available, but also that participation is open and linkages between, and provenance of, research objects are clear, open and sustainable.

To promote the scholarly commons we provide core principles that participants and stakeholders can ascribe to. Thus, the scholarly commons is an agreement among knowledge producers and users.

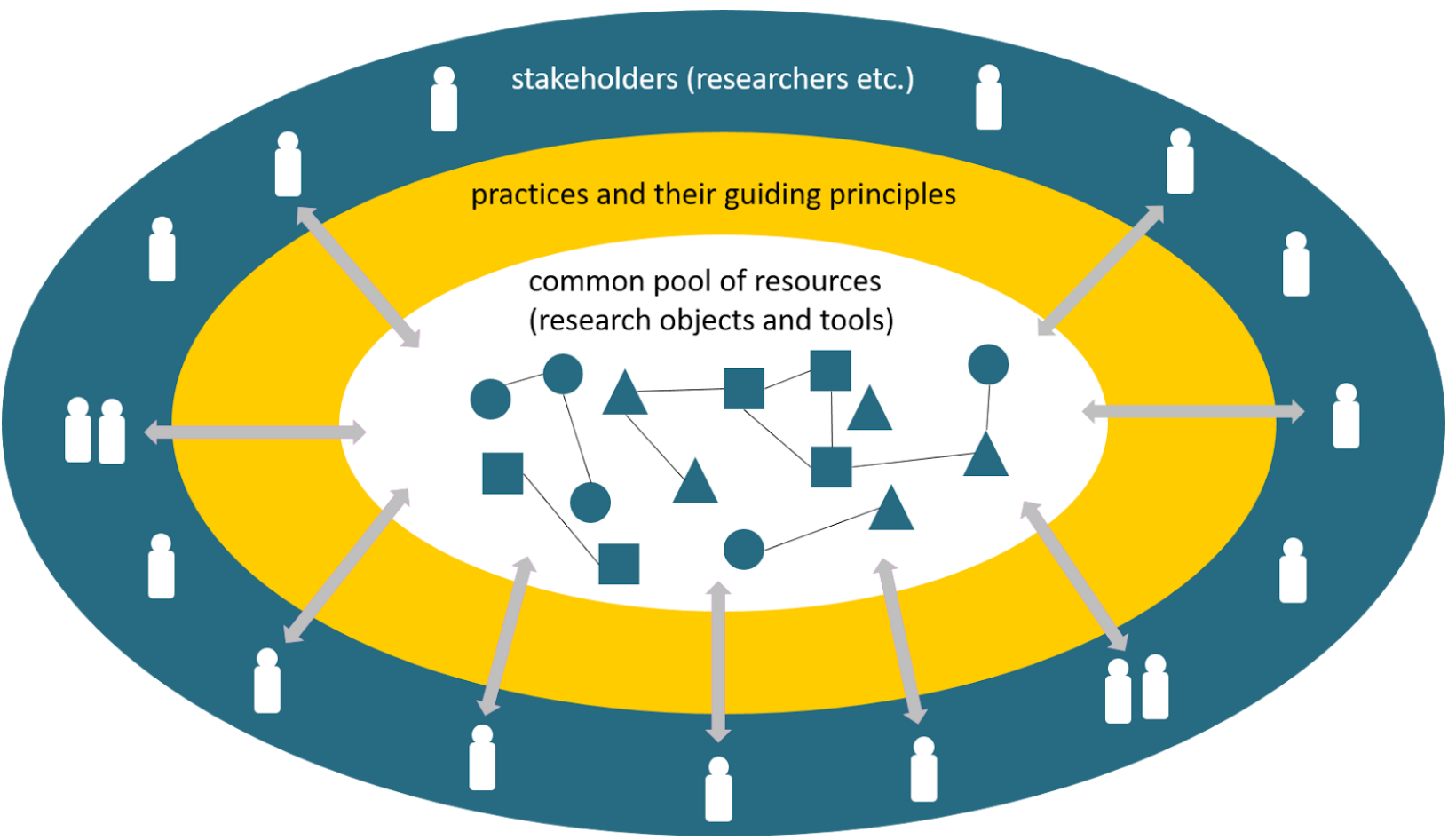


Figure 1. The scholarly commons, which includes the stakeholders involved in research, the principles that guide their practices, as well as the tools they use and the research objects they create, share and reuse.

In taking the commons idea as a starting point, the scholarly commons we envision differentiates itself from other initiatives in research communication that use the term "scholarly commons" or "commons": in some cases as a name for their institutional repositories and in other cases for a field specific platform (e.g. the NIH Commons or Humanities Commons in the US).

The scholarly commons may be thought to align with the ideas of Creative Commons and the P2P Foundation and in many ways that is true, but, as we shall argue, the scholarly commons is specifically focused on research.

The scholarly commons idea is the result of a programme coordinated by FORCE11 through its Scholarly Commons Working Group, that started work in October 2015 after receiving a grant from The Leona M. and Harry B. Helmsley Charitable Trust Biomedical Research Infrastructure Program. FORCE11 is an organisation seeking to improve research communication by stimulating innovation, by formulating standards and by fostering consensus about those among all stakeholders. The idea of the scholarly commons is accompanied by the launch of the scholarly commons website that we are positioning to inspire, connect and discuss the many initiatives out there. The scholarly commons working group also started to discuss inclusivity perspectives that should continuously inspire these principles and started providing guidelines in the form of decision trees that help putting the principles into practice.

This paper outlines the backgrounds of the idea of the scholarly commons (section 2), the various considerations that play a role in defining it (sections 3 and 4), the principles (section 5), the degrees of freedom interpreting those principles (sector 6), the broader landscape of ideas and charters that it fits into (section 7) and finally presents a call for action (section 8).

\section{Starting from scratch - functionalities or principles?}

"You never change things by fighting the existing reality. To change something, build a new model that makes the existing model obsolete."-Buckminster Fuller [attributed]

The Scholarly Commons program at FORCE11 had several antecedents, including an earlier version of the Scholarly Commons group that explored commonalities between the humanities and the sciences. But the program really took shape after the FORCE2015 meeting held in Oxford in January, 2015. The impetus was provided by two threads at this meeting: the first, was the presentation on 101 Innovations in Scholarly Communication, by Jeroen Bosman and 
Bianca Kramer. This important work not only cataloged some of the new tools and platforms available but also their use within the scholarly community.

The second was a $1 \mathrm{~K}$ Challenge idea put forward by Sarah Callaghan from the Centre for Environmental Data Analysis, STFC, UK:

\section{Re-do from start - what would research communication look like after a clean start? Research communication carries with it the weight of 350 years of tradition, still using workarounds for technological limitations from many centuries ago. What would research communication look like if we threw everything out and started again, given current technologies? I'd propose bringing people together for a day's workshop where we'd start with a blank page, and design something different that would meet the needs of researchers and users of research. At worst we'd have a better understanding of what it is research communication needs to be able to do. At best, we might even come up with something revolutionary!}

Sarah's challenge and the 101 Innovations in Scholarly Communication work together provided the perfect framing for the scholarly commons program because it allowed us to think across the landscape of scholarly communications as a whole, rather than addressing only a single aspect of it. Our first goal was to determine if there was a shared vision. Did all organizations, platforms and individuals dedicated to transforming scholarly communication share a common idea of what we were transforming it into? The second goal: if the answer was yes, how close were we to realizing it? That is, among the dozens of declarations, charters, manifestos that have been produced since FORCE11 launched, was there a coherent vision that could be articulated that would allow us to communicate effectively and take stock of where we stood with the hundreds of tools that had been created or proposed?

To explore this idea, FORCE11, with support of the Helmsley Foundation, held a workshop in Madrid in February 2016: ' Re-imagining research communications' (Kramer et al. 2016). Fifty participants were brought together with the following charge:

The scholarly system has vanished. The forms of communication that were valid just a moment ago are now part of history. The contracts (international and individual) have ceased to exist. All the universities, publications and job titles are no longer in effect. The good news is that this is not an end: We have gathered this very group here to re-start the whole system, and this is a chance to do it better. You can use the same amount of money and technology that there was available before, but use it better. Another good news is that your intelligence, knowledge and creativity have not vanished. We will need those qualities today. We are happy you made it to this emergency meeting. Because of this situation, the scholarly world faces the question: Now that we have to start from a blank slate, how do we want to organize ourselves? How could we create our communication anew? 
Note that in the framing for this meeting, we neatly sidestepped two of the thorniest issues that usually face any discussion of new modes of communication:

1. Who is going to pay for it?

2. What is the incentive for (me, you, they) to participate?

The answer to 1 is "the same people who are paying for it now, they are just going to be paying to make scholarly artifacts commons compliant: open, FAIR, citable - and not closed, unFAIR and woefully under-attributed." The answer to 2 is "you are designing the system to serve the needs of scholarship, scholars and society; incentive systems that are put in place will drive the community towards better scholarship and greater service to society, not away from these goals." In a previous mini-version of such a workshop in Dagstuhl, held in July 2015, we found that by dispensing with these two issues from the getgo, participants were free to explore what it meant to serve the needs of scholarship and society and not try to fit things into our current skewed reward system.

After Madrid, the answer to our question: "Are we ready to define the scholarly commons?" was an emphatic "maybe". Through the exercises, it was clear that there did, in fact, exist a general sense of how a scholarly commons should operate and a set of principles that would govern the production of scholarly objects.

As with the Dagstuhl meeting, the shape of the commons, although largely amorphous, was envisioned to work rather like the open software community. That is, the production of products would be open, collaborative and iterative with clear versioning and provenance. As per Carole Goble's maxim from the FORCE11 Beyond the PDF 2 conference (Goble 2014): we wouldn't publish, we'd release. Of course, the commons would encompass the entire spectrum of scholarly objects and activities: narrative, code, data and discussion. All entities within the commons, including its products, the people who produce and consume them would have digital identities that would make them FAIR: Findable, Accessible, Interoperable and Reusable.

The commons would be reflexively open. Limits to openness were to be expected where safety or ethical issues were a concern, but the expectation was that all objects in the commons would be designed specifically for maximal sharing, machine-based access, and reuse without restriction.

The commons would have publishers, but a publisher would be considered any entity that makes scholarly objects available according to the principles of the commons. Integral to this function would be ensuring that objects are open, FAIR and citable. With FAIR and citable comes the idea of persistent as well. Thus, publishers would be charged with ensuring the long term persistence of scholarly objects.

The commons envisioned would have additional roles, particularly in the form of what one group coined "knowledge workers". Knowledge workers would be those who worked with scholarly objects to make them better or to adapt them for different purposes. In our current system, we recognize these knowledge workers as editors, reviewers, curators, communications experts. As 
scholarly objects are created and passed through different spaces within the commons they would be touched by many individuals and algorithms. All of these activities would be attributed, so that a work would come with a list of "credits", similar to movies, reflecting all the different types of contributions across its lifecycle.

Some knowledge workers would become known by their ability to create collections of value to others or by their particular skills.

The commons as envisioned therefore would be inclusive:

- To scholarly objects: all scholarly objects would be welcomed in the commons, barring ethical violations, and all objects and actions on these objects in the commons would be attributed throughout the lifecycle of an object

- To commercial and non-commercial; academic and non-academic entities. Knowledge workers would be recognized as valuable contributors and operate in the commons the same as scholars.

What were the spaces envisioned for the commons? One group developed the idea of a food-based metaphor. The commons should provide spaces for harvesting of ingredients, a pantry where they are organized, a food-preparation and testing kitchen, and a restaurant, where mature dishes are presented for consumption and broader feedback. That scholarly works are built from other works is central to scholarship, and that their creation involves an iterative process of refinement, many dead ends, and aborted efforts is a fact. The raw ingredients come from the world around us and the wider web. The pantry contains the existing works in the commons built from these ingredients. The commons invites everyone into the kitchen where these processes occur, and they are welcome to sample and engage with the chefs, but we know that this is a preparation area and that the counters and floors might be messy and some dishes not fully realized.

This metaphor essentially embodies the scholarly life cycle and the foundational inspiration 'Don't publish. Release!' (Goble 2014), while respecting the very human tendency to want to be judged on our finished products and not early drafts.

The focus on scholarly objects took up a surprisingly small percentage of the workshop exercises and was not explored as fully as some of us expected or, in truth, would have liked. It was almost as if the technical details of how these objects should be produced were not the most important consideration at this point. Rather the bulk of time was taken up by considering the people in the commons (i.e., the commoners). Perhaps because of the diversity of perspectives that were present and a meeting structure that encouraged interactions among these diverse perspectives, a common vision was a little more elusive. Some discussions focused on how people should behave in the commons, e.g., expectation of service, new roles for commercial entities, publishers and journals. But a large number of discussions were devoted to how people should be treated in the commons. A strong feeling that people should be able to develop a reputation in the commons by what they did rather than by where they were located was strongly expressed. The idea that everyone should be represented by a 5 
minute pitch (an "API to oneself") in the form of a video or graphic, rather than just a set of credentials was proposed. More senior participants wanted to be able to identify talent and be able to assemble strong research teams that could be devoted to a single project as needed. But more junior participants, and those currently from diverse geographic regions were more concerned about access and equanimity: how are scholarly works made accessible to all, and how do those who are not at top tier universities in the US and Europe get their work noticed?

When considering the outcomes from this workshop in all its messiness and diversity, what emerged strongly was that the scholarly commons was based on values. The technical details, the business models, the incentive system, all should reflect the purpose of the commons: to make the fruits of research and scholarship better and available to all who need or want them. To do this, we need to recognize that people are the most important "scholarly objects" in the commons.

\section{From inclusiveness to a minimal viable prototype?}

The first workshop had yielded, through a bottom-up process, one and a half dozen principles that touched upon open, equitable, sustainable, and research- and culture-led scholarship. But could the principles in this form indeed be used as guiding principles for what the scholarly commons should look like in practice? In our second workshop, in September 2016 in San Diego, we aimed to put that question to the test by asking representatives from various communities to reflect on the principles and assess how current practices and research infrastructure hold up against them. What pieces do we have? Where are we lacking inclusivity, expertise, infrastructure, tools? Where are their significant barriers? What opportunities exist for the community to help fill these gaps and how can we create community buy-in around proposed solutions?

It soon became apparent that there were still major issues to address, both regarding the principles themselves and regarding the inclusivity of the process through which they were derived. In the end, a large part of the workshop was devoted to addressing these concerns. At the same time, the concept of a minimal viable prototype (MVP) for the scholarly commons was explored.

\section{Principles}

Due to the bottom-up process that was employed in the Madrid workshop, the principles generated there reflected a range of important issues, but not one coherent underlying vision. At the San Diego workshop, one such underlying vision was developed based on Mertonian norms (Wikipedia contributors 2017), starting from the assumption that to re-engage knowledge with wider communities, we need a return to basic principles that support effective scholarship as a common good. The classic Mertonian norms of universalism, communalism, disinterestedness 
and organized skepticism were mapped onto the idea of the scholarly commons to arrive at 4 general aims for effective scholarship:

- universalism: don't discriminate, but do evaluate (equitable)

- communalism: don't enclose, but do steward (open/FAIR)

- disinterestedness: don't obscure conflicts of interest, but do work to reduce bias (transparency)

- organised skepticism: don't circumvent scrutiny, but create [private] space for creativity (research-culture-led)

This approach basically starts from a coherent view of norms for scholarly communication, and careful evaluation would be needed to see how many of the issues brought forward in Madrid would be covered by these a priori norms and vice versa.

Daniel O'Donnell went one step further in his attempt at synthesizing the principles. From the principles gathered in Madrid, he tried to construe a cohesive set of 'principled principles' - a set of statements from which everything in our work so far would need to be derived or implied (O'Donnell 2016a; O'Donnell 2016b). As a result, he defined the scholarly commons is an agreement among knowledge producers and users that research and knowledge should be freely available to all who wish to use or reuse it, participation in the production and use of knowledge should be open to all who wish to participate, and our practices should be such that there are no systemic barriers and disincentives to prevent either such free use or open participation.

In order to meet this definition, then, a number of rules need to be followed. Together, this encompasses most, if not all, of the issues brought forward in Madrid. After the San Diego workshop, a subgroup of the Scholarly Commons Working Group used this framework to develop the next iteration of the principles, which is presented in section 5: Principles.

\section{Inclusivity}

So far, North American and Western European perspectives dominated the work of the Scholarly Commons Working Group and its draft principles. In this way, the Scholarly Commons initiative did not include enough diverse and important perspectives. During and after the San Diego workshop, these issues were discussed extensively and a working group was formed to create recommendations for improvement and best practices for the work on the scholarly commons and other initiatives to improve scholarly and scientific communication. Through a series of open community calls, asynchronous collaboration, and an in-person workshop, this subgroup hopes to contribute to and advance the conversation about how a scholarly commons could "meaningfully be built for and by researchers in the global south" (Hathcock 2016) and other communities. 


\section{Minimal Viable Prototype (MVP)}

While acknowledging the issues described above, we also used the second workshop to explore the potential of the principles to identify commons-compliant research workflows, and a so-called minimal viable prototype (MVP). This combined systematic checking of tools/platforms for compliance with the principles with an attempt to define what research practices would be essential in the commons, and matching those to existing commons-compliant tools and platforms.

This first stab at looking at possible practical implementations of the commons revealed several things. It underscored the need for a cohesive set of principles that can also be operationalized, i.e. translated into practical requirements for tools/platforms and practices. It also demonstrated that many commons-compliant practices and tools/platforms already exist, but in relative isolation. The challenge is how to bring them together, both at a conceptual level (what are the motivations and barriers for researchers to engage in these practices?) and at a technical level (availability and interoperability of platforms and tools).

A minimal viable prototype could then be seen as a demonstration of the infrastructure needed to practice research in the scholarly commons - either as set of standards or as concrete example workflows using a selection of commons-compliant tools and platforms. It could even be a new platform altogether, encompassing all functionalities needed for the scholarly commons. By identifying the 'low hanging fruit' that would constitute a minimal viable prototype (see Figure 2 for a hypothetical example), it could serve as an easy entry-point into a way of doing research in the scholarly commons. Its infrastructure could subsequently be used, refined and grown to accommodate a broader variety of workflows and communities.

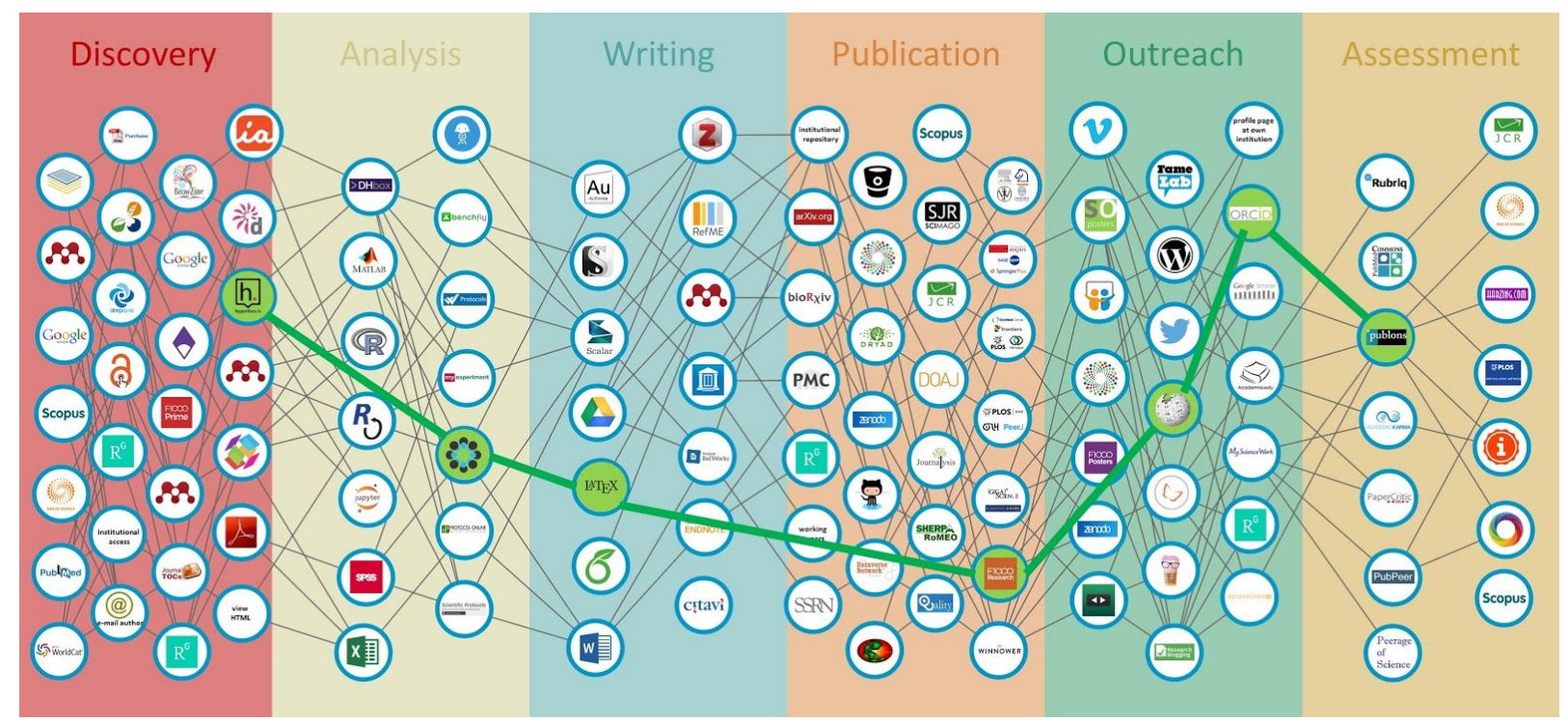

Figure 2. Hypothetical example of a minimal viable prototype of the scholarly commons, consisting of existing tools/platforms used together in a research workflow. Image used (and partially generated) at the San Diego workshop.

In thinking about the implementation of the scholarly commons, it is important to account for 
disciplinary and (inter)cultural differences. One size does not fit all, and communities will need to define their own interpretation and implementation of the principles, practices and platforms of the commons, both at a human and technological level.

One very concrete way of providing guidance to researchers and other stakeholders in scholarly communication on how to practice commons-compliant scholarship could be by developing decision trees. These could guide people in their choices for commons-compliant research practices and how to implement them, and could be adapted by and for different disciplines and communities. Developing a framework for such decision trees has been the focus of a third subgroup of the Scholarly Commons Working Group following the San Diego workshop.

A fourth subgroup has since convened to explore enabling technologies and infrastructures for the scholarly commons from a more conceptual viewpoint and with the mindset that, although communities will need to define their own interpretation and implementation of the principles, practices and platforms, the scholarly commons will be most effective if it is realized, on some level, in a coordinated way. The focus of this working group is on finding or creating working technical implementations, based specifically around the principles of the scholarly commons, that will give us an easier way forward than our current set of tools in helping to build the culture of the scholarly commons. Notwithstanding the technical focus of this group, this group is calling for the participation of people of various worldviews and backgrounds, to be able to converge upon viable alternatives and solid, sustainable solutions.

\section{Principles}

The principles of the scholarly commons set the norms for activities in the scholarly commons. Derived through a process of bottom-up inception and subsequent synthesis, they start from the vision that scholarly communication needs to be as open and participatory as possible, and lay out the rules needed to achieve that (Fig 3, Table 1). The commons as we envision it is about scholarly objects being open, FAIR and citable, but also about participation, independence of external reward systems, and independence of specific (technical) systems, platforms and business models.

The principles can be used as (1) a framework for an agreement between researchers, funders, publishers, librarians, citizen scientists and others active in science and scholarship on how to make research open and participatory for anyone, anywhere, (2) a guide to decide how to practice and support research, and (3) an eventual basis for badging of activities as commons-compliant, by individuals and organizations. 


\section{The scholarly commons is an agreement among knowledge producers and users that}

\section{research and knowledge should be freely available to all who wish to use or reuse it (open, FAIR and citable)}

\section{participation in the production and use of knowledge should be open to all who wish to participate}

\section{there should be no systemic barriers and disincentives to prevent either such free use or open participation}

Figure 3 High-level principles of the scholarly commons. The principles start from the vision that scholarly communication needs to be as open and participatory as possible, and lay out the rules needed to achieve that.

Principles of the scholarly commons (version 0.1 .1 )

P1. The scholarly commons is an agreement among knowledge producers and users.

This means that:

- The commons is developed by its members through their practice

- There is global commitment and participation in the commons' long-term viability and preservation

P2. Research and knowledge should be freely available to all who wish to use or reuse it.

This means that:

- The commons is open by default

- Scholarly objects and content in the commons is FAIR: findable, accessible, interoperable and reusable by humans and machines 


\section{P3. Participation in the production and use of knowledge should be open to all who wish to participate.}

This means that:

- The commons welcomes and encourages participants of all backgrounds

- The commons is open to all participants who accept its principles

\section{Rules}

In order to effect these principles, participants in the commons agree that:

\section{R1: The rewards for participating in the commons are access, opportunity and attribution}

This means that:

- Provenance of objects in the commons should be transparent and persistent

- The commons has no intrinsic hierarchies, rankings, or reward systems

\section{R2: The commons is agnostic regarding form and technology}

This means that:

- The commons exists independently of technology, funding, and business models that support and enable it

- The commons accepts all contributed objects that adhere to its guidelines on an equal basis regardless of form, genre or approaches

R3: (Use of) external systems or technology, including reward systems, must not harm the commons.

This means that:

- The form research is disseminated in is determined by the needs of the research itself

- All activities and outputs that take place in in the commons remain in the commons

Table 1 Principles of the scholarly commons, version 0.1.1 (FORCE11 Scholarly Commons Working Group 2017)

The principles are ambitious: they describe what to strive for, what that means in more concrete terms, and what is important in order to get there. At the same time, the principles are not confining: individuals and organizations can differ in the commons-compliant practices they 
adopt and in the speed at which they do so. For example, while scholarly objects should be open, FAIR and citable, there may be reasons for researchers (especially in social sciences and humanities) to remain anonymous, and the commons should accommodate that.

Also, the commons can thrive alongside other systems of scholarly communication - in so far as these do not prohibit participation in the commons. For example, the commons does not deny or preclude the existence of external reward systems (e.g. criteria for promotion and tenure), but those should not limit making research openly available and accessible.

The principles themselves do not describe what the scholarly commons should look like and how it should be organized and governed. That is up to participants to decide, using the principles to guide their practices. This can involve choosing existing systems and platforms to carry out and disseminate research, as well as creating new solutions.

The principles are a beginning, not an end: they can only become meaningful when individuals and organizations in various disciplines and cultures critically consider them and try to answer the question: are we ready to define the scholarly commons?

For this, we want to put the principles before various communities, across disciplines, geographical areas and stakeholder groups, and ask them how these principles relate to their practice. Can they see the principles working in their specific situation(s)? We also want to involve the community to check the set of principles against the most important existing charters, and formulate use cases to check whether the principles uphold in those cases.

\section{Commons interpretation, from maximal to grey}

When fostering change and trying to realize a scholarly commons the question arises what is considered a positive contribution and what not. We would like to see growth of the amount of research practices and research objects and comply with the principles of the commons.

Compliance is asked from stakeholders, tools, practices and research objects. The function of checking compliance of tools, research objects and practices is to make sure that the pool of compliant elements that can be used and go well together is clear and also to promote making these objects, practices and tools compliant. Compliance here pertains to all the principles, as far as relevant for the specific object, tool or practice.

A researcher can have some commons compliant research objects and can carry out some compliant practices and use some compliant tools, whilst others are still non-compliant or only compliant with some of the principles. A publisher may have a fully compliant tool, but still non-compliant content. A funder may have some grants with regulations that are compliant and others that are still not. In other words, there can be shades of grey, not just black and white. This is notwithstanding the fact that stakeholders can badge themselves as striving for 
commons compliance. Stakeholders using such a badge proclaim their intention to contribute to the commons as much as they can.

Using this interpretation, the commons itself is a pool of compliant tools, practices and objects, supported and created by a community that hopefully publicly proclaims their intentions to contribute to the commons and used by anyone who wishes to use it.

Knowing that research is a global effort involving hundreds of millions of researchers, students and people supporting research, this means that the (global) scholarly commons is not necessarily a tightly organized and governed community around a common resource pool. Also that resource pool can be a very distributed one, its configuration reflecting present and future differences in the scholarly information landscape across disciplines, cultures and language areas. Consequently governance of the scholarly commons might only go so far as checking compliance of tools and practices and stimulating continuous discussion among stakeholders proclaiming themselves to work towards commons compliance. Thus, the scholarly commons is not necessarily a full 'maximal' commons (as in: one integrated system that is governed as a whole). However, we expect that working towards the scholarly commons will imply that compared to the current situation there will be at least some convergence of types of practices and tools used and also increase of strictness on what ways of working and what type of tools and output are welcomed given the principles that we have. The hypothetical path towards that is depicted in figure 4 that shows four scenarios: apart from the current situation and a full maximal commons, also a distributed but strict situation (where all funders, publishers, and institutions implement the principles) and an integrated but lenient solution (call it ResearchGate). 


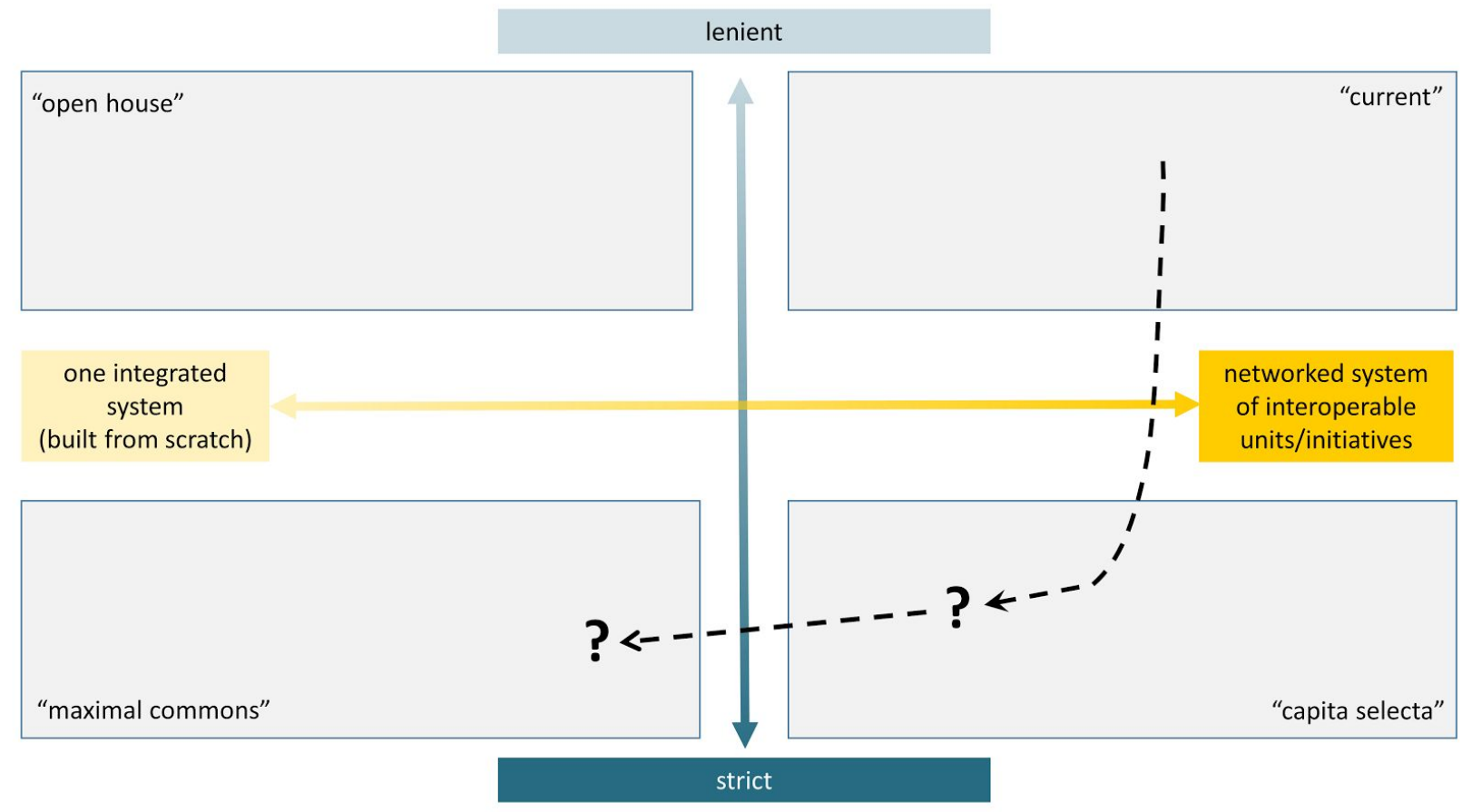

Figure 3. Four scenarios for the organization of scholarly communication along two dimensions: adherence to scholarly commons principles and integration of practices, tools and platforms. Dashed line: hypothetical paths towards a scholarly commons.

\section{Not in a vacuum: 7 dozen declarations and 1 dozen initiatives with commons aspects}

Trying to improve scholarly communication is not new, and neither is the idea of commons. Hundreds of tool builders, publishers, societies, funders and individual researchers have taken action to make scholarly communication more efficient, open and/or reproducible. Important as they are, most of these initiatives are scattered, applied at only one set of journals, or accepted in just one discipline or country. With the digital revolution we have also seen initiatives trying to tackle issues more fundamentally. This has resulted in least 85 charters that were created since 1986 addressing scholarly communication and knowledge creation (see this preliminary list of charters). Their scope is often still limited, focussing for example on either access, or evaluation or technical standards (excepting the Vienna Principles that address most issues). The charters mostly do aim at global usability and acceptance. However, we must acknowledge and question the fact that almost without exception they originate from US and Western-European individuals and organizations, and some of them are indeed only available in English. It is encouraging that some of the most important charters, illustrated in Figure 4 and summarized in Table 2, have 
gained widespread international acceptance. Also, they are well known beyond the small circle of research communication professionals, i.e. among researchers themselves, either as the charter itself or through the effects the charter had on real practices. The effects of the charters are complex and hard to gauge. Overall one could say that realization of changes the charters propose is not marginal anymore but on the other hand has not become mainstream either. Open Access, altmetrics, FAIR data and more are widely available and practiced, but not the norm, yet.

Thus, the current proposal for a scholarly commons is in many ways inspired by and credits those charters but can be said to be different from other charters and declarations in its comprehensive approach in terms of issues, and in its commons-based foundation. It fosters changes across the research workflow by all stakeholders involved, not just researchers.
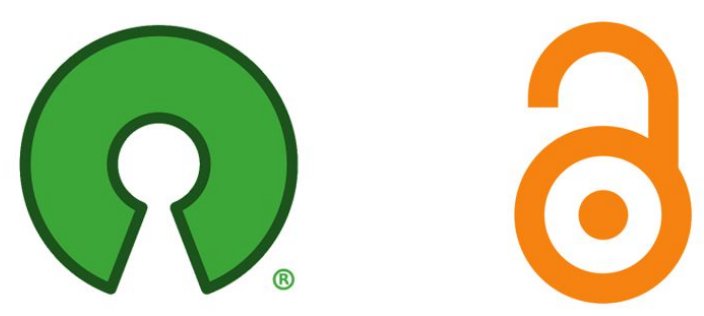

\section{altmetrics}

San Francisco

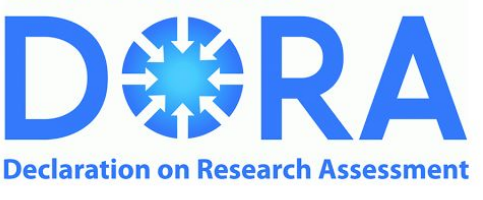

Declaration on Research Assessment

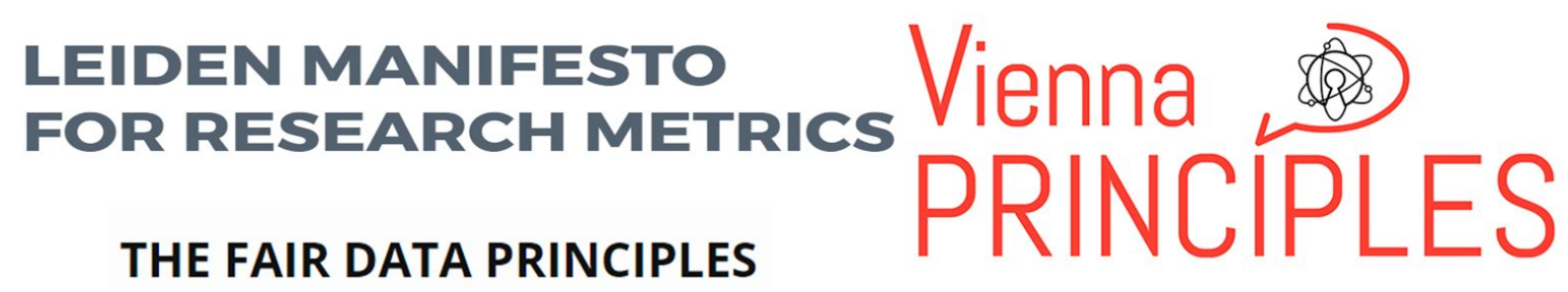

The Open Definition

Figure 4. Some important scholarly communication charters (see Table 2 for list with characteristics) 


\begin{tabular}{|l|c|c|c|c|c|c|}
\hline CHARTERS & $\begin{array}{c}\text { inclusivity / } \\
\text { participation }\end{array}$ & $\begin{array}{c}\text { access / } \\
\text { copyright }\end{array}$ & $\begin{array}{c}\text { evaluation } \\
\text { / funding }\end{array}$ & $\begin{array}{c}\text { reprodu- } \\
\text { cability }\end{array}$ & $\begin{array}{c}\text { linking / } \\
\text { standards }\end{array}$ & outreach \\
\hline Open source definition & $\checkmark$ & $\checkmark$ & & & & \\
\hline Berlin declaration & & $\checkmark$ & & & & \\
\hline Open definition & $\checkmark$ & $\checkmark$ & & & $\checkmark$ & \\
\hline Altmetrics Manifesto & & & $\checkmark$ & & & $\checkmark$ \\
\hline SF DORA & & $\checkmark$ & $\checkmark$ & & & \\
\hline$\underline{\text { Leiden Manifesto }}$ & & & $\checkmark$ & & & \\
\hline FAIR data & & $\checkmark$ & & $\checkmark$ & $\checkmark$ & \\
\hline Vienna Principles & $\checkmark$ & $\checkmark$ & $\checkmark$ & $\checkmark$ & $\checkmark$ & $\checkmark$ \\
\hline
\end{tabular}

Table 2. Selection of important scholarly communication charters compared by the issues they address.

In its goals, the scholarly commons overlaps with advocacy and concrete creation of standards and tools by many organizations, including FORCE11 itself. It draws upon these efforts as both the inspiration for and concrete aspects of the commons. Comparing the proposed scholarly commons to a number of such other initiatives (illustrated in Figure 5 and summarized in Table 3 ) shows that currently the scholarly commons is modest: principally a set of rules and principles with ideas on how they could work, in practice as well as from an inclusivity perspective, and also describing the collective outcome of practices based on those principles. But that common pool of resources is not governed or managed by the current scholarly commons initiative. There is no dedicated hard infrastructure and though there may be a nascent community, there is no formal membership. In its approach the current scholarly commons has most in common with the Peer2Peer Foundation, but of course it is limited to research and scholarly practices. 


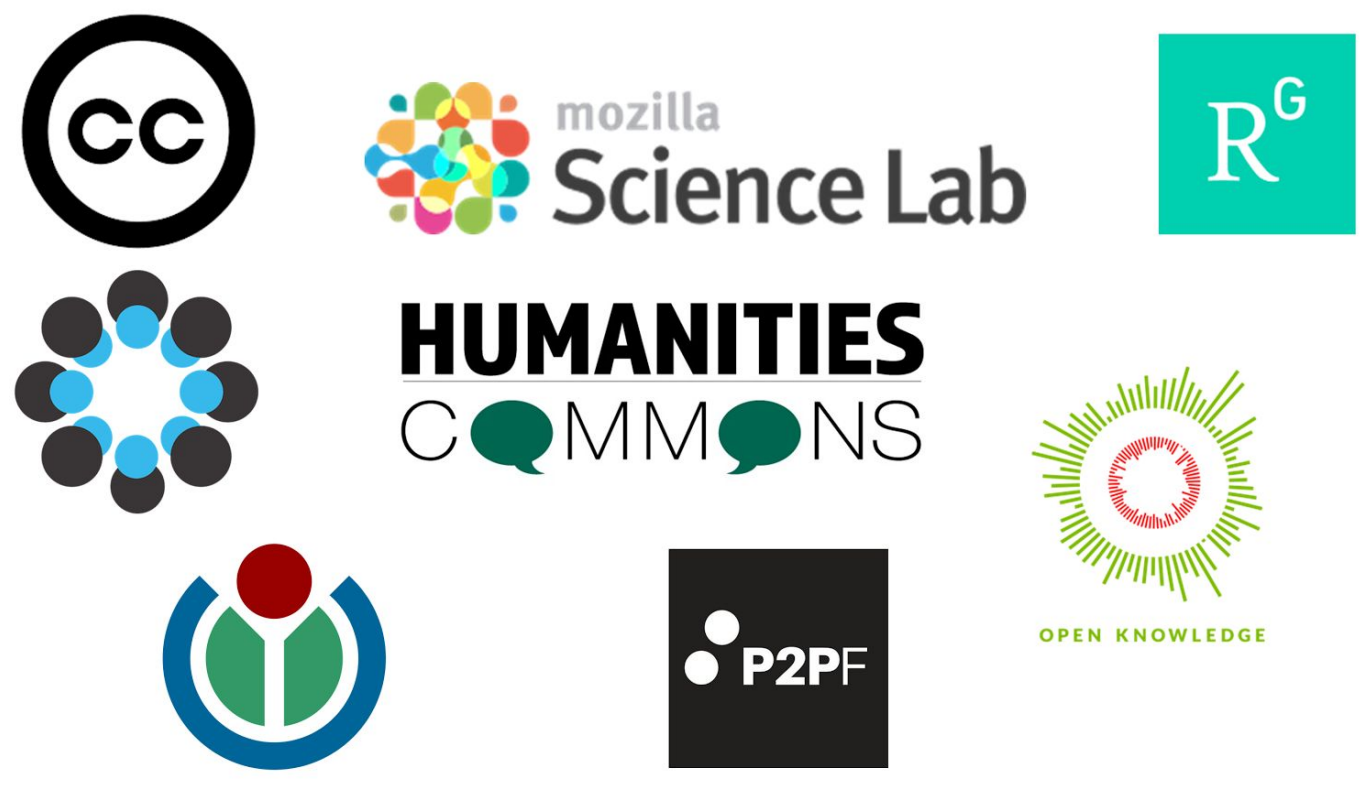

Figure 5. Some initiatives and organizations related to scholarly commons (see Table 4 for list with characteristics)

\begin{tabular}{|l|c|c|c|c|}
\hline INITIATIVES & $\begin{array}{c}\text { manages 'own' } \\
\text { common } \\
\text { resources pool }\end{array}$ & $\begin{array}{c}\text { social protocols/ } \\
\text { rules/principles/ } \\
\text { soft infra }\end{array}$ & community & infrastructure \\
\hline Scholarly commons (proposal) & & $\checkmark$ & $?$ & \\
\hline Creative Commons & & $\checkmark$ & $\checkmark$ & $\checkmark$ \\
\hline Mozilla Science & $\checkmark$ (enclosed) & & $\checkmark$ & $\checkmark$ \\
\hline ResearchGate & $\checkmark$ & $\checkmark ?$ & & $\checkmark$ \\
\hline Open Science Framework (COS) & $\checkmark$ & & $\checkmark$ & $\checkmark$ \\
\hline Humanities Commons & $\checkmark$ & $\checkmark$ & $\checkmark$ & $\checkmark$ \\
\hline Open Knowledge International & & $\checkmark ?$ & & $\checkmark$ \\
\hline Wikimedia & & $\checkmark$ & $\checkmark$ & \\
\hline Public Knowledge Project & & & & \\
\hline Peer 2 Peer Foundation & & $\checkmark$ & $\checkmark$ & $\checkmark$ \\
\hline
\end{tabular}

Table 3. Scholarly Commons compared to related initiatives and organizations. 
Overlooking the landscape of charters and commons oriented and comparable organizations it is clear that the commons approach and the comprehensive perspective add value, but to be effective it should seek to collaborate and align with other initiatives and intertwine ideas. The form of that collaboration probably depend on the way the scholarly commons will operate. What are the mechanics and practices of the scholarly commons?

\section{Badges for starters, decision trees as mains, but be welcome in the kitchen}

The commons can only grow and become meaningful when individuals and organizations in various disciplines and cultures contribute to its process, principles and practices. So what can you do today to help make science and scholarship become more open and participatory? There are a number of ways we invite researchers, funders, publishers, librarians, citizen scientists and others to help shape the scholarly commons and start making a difference. You are welcome in the kitchen (www.scholarlycommons.org, Figure 6). Start now!

\section{The Scholarly Commons}

INCLUSIVITY

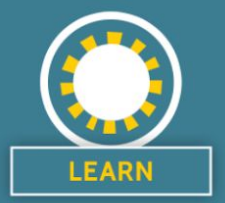

PRINCIPLES

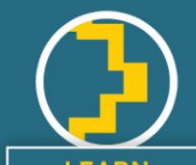

LEARN

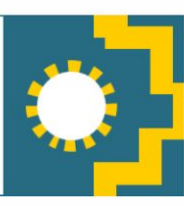

PRACTICE

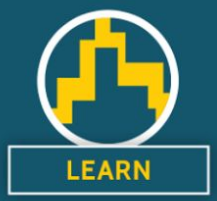

The scholarly commons is an agreement among researchers and other stakeholders in scholarly communication to make research open and participatory for anyone, anywhere. It is not another sharing platform, but a set of principles, concrete guidance to practice, and actions towards inclusivity of diverse perspectives from around the globe. Though a Forcell initiative, the scholarly commons is owned by no one, to be realized. used and contributed to by all.

\section{START NOW!}

Calling all researchers, funders, publishers, librarians, citizen scientists and you.

Figure 6. English-language version of Scholarly Commons website https://scholarlycommons.org (retrieved August 29, 2017) 
Areas to get active in:

\section{Inclusivity}

Contribute to the work of the subgroup on inclusivity to critically examine how inequalities and biases are reflected in scholarly communication practices and recommendations, and how a scholarly commons could be built to meaningfully reflect and advance the practices, innovations, and goals of different perspectives. Join the discussion, share your thoughts and best practices, and incorporate recommendations from the group in your daily practice, especially when organizing groups and group activities around the future of scholarly communication.

\section{Principles}

Consider how the principles relate to research practices in your community. Can you see the principles working for you? Which principles do or do not apply to your specific situation? We also want to involve the community to check the set of principles against the most important existing charters and declarations on open science and scholarship and research conduct, to see where the scholarly commons differs from what these charters aspire to, and where the goals of different organizations align.

\section{Practice}

Explore ways to practice science and scholarship in the scholarly commons

\section{- Decision trees}

Use decision trees to help you decide how to carry out and support research in a commons-compliant way right now, e.g. by getting guidance on sharing data and choosing a data repository. You can also adapt decision trees for use in your own community, or create new ones using our workflow that we tried to make as commons-compliant as possible.

- Enabling technologies and infrastructures

We are having discussions about enabling technologies in a community fashion in order to have the unity in approach and the support and consensus needed to arrive at technical standards, working implementations, and better solutions, compared to what is already available. If you have an idea for a better way of doing research, come share it with us! We need supportive technologies and infrastructures that will enable the culture of the commons to flourish.

\section{- Badging}

We are exploring ways for individuals and organizations to badge their activities as commons-compliant, e.g. by listing tools, platforms and practices that are compatible with open and participatory scholarship, or by providing a decision tree to self-assess commons-compliance of your research activities and the platforms and tools you use or support. Badging your activities can be a way to declare your intentions as an individual 
or an organization and say "I am committed to certain practices across my scholarly practice so don't ask me to do things contrary to them".

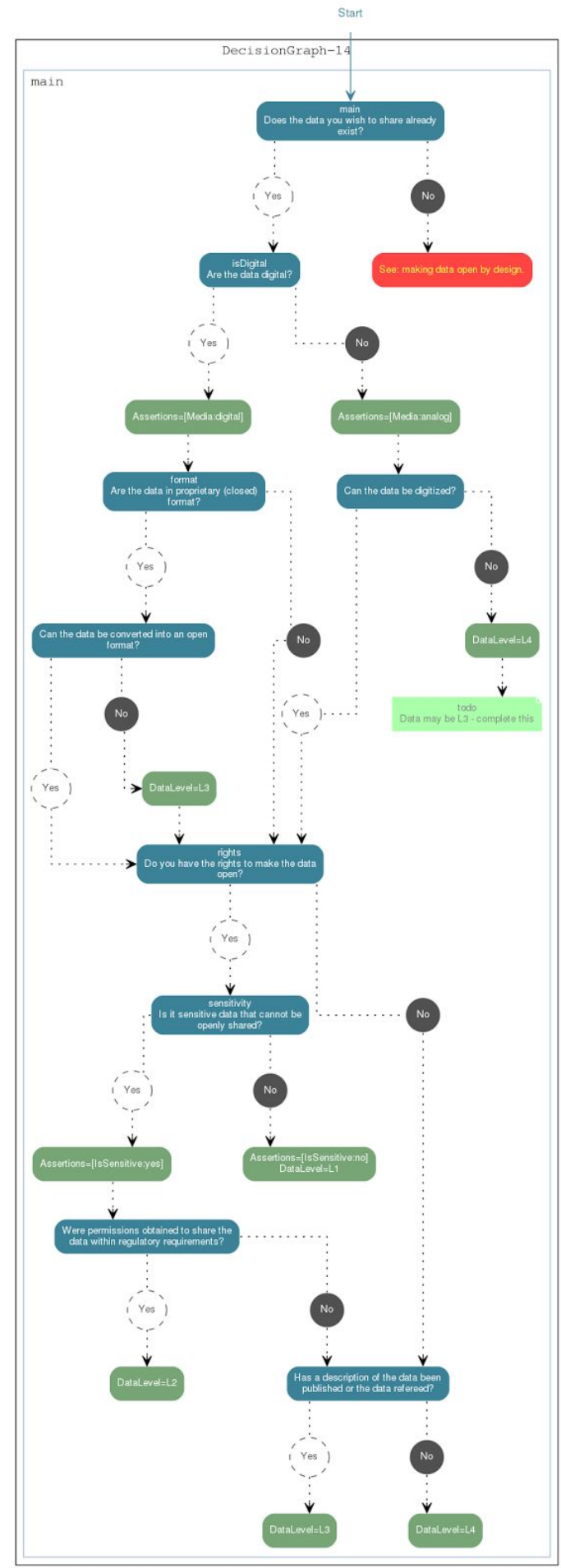

Figure 7. Example of a scholarly commons decision tree on making data open 


\section{Share your story}

How do you practice research in a commons-compliant way? What parts of your research workflow are open, and how do you encourage others to participate in your research? Are you a citizen scientist collaborating with academic researchers on a science project from start to finish? A librarian, publisher or funder stimulating inclusivity and sharing and reuse of research output, from grant proposal to post-publication peer review? Your story might inspire others to follow your example, take the next step and work together towards more open and participatory scholarship.

\section{Keep the conversation going}

We still have many questions around the scholarly commons: regarding processes to be as inclusive as possible, the definition of the principles, and the implementation of the scholarly commons in practice as a collaboration between researchers, funders, publishers, librarians, citizen scientists and anyone else with an interest in scholarly communication. We are starting this conversation by posting this preprint now, with the aim of revising and publishing a subsequent version at a later stage, incorporating the feedback we get. We also want to work in tandem with other organizations promoting open and participatory scholarship, to mutually reinforce our efforts. Please join the conversation, ask questions and add your comments at our discussion forum. Are we ready to define the scholarly commons?

\section{References}

Berners-Lee, T.J., 1989. Information management: A proposal. No. CERN-DD-89-001-OC. http://cds.cern.ch/record/369245/files/dd-89-001.pdf [Accessed August 21, 2017].

Bosman, J. \& Kramer, B., 2015. 400+ Tools and innovations in scholarly communication. Google Docs. Available at: http://bit.ly/innoscholcomm-list [Accessed August 21, 2017].

Conley, J.P. \& Wooders, M., 2009. But what have you done for me lately? Commercial Publishing, Scholarly Communication, and Open-Access. Economic analysis and policy, 39(1), pp.71-88. https://doi.org/10.1016/S0313-5926(09)50044-6.

FORCE11 Scholarly Commons Working Group, 2017. Principles of the scholarly commons, version 0.1.1. https://doi.org/10.5281/zenodo.569952.

Goble, C., 2014. Don't publish. Release! FORCE11. https://www.force11.org/presentation/dont-publish-release [Accessed September 13, 2017].

Hathcock, A., 2016. Making the Local Global: The Colonialism of Scholarly Communication. At The Intersection. 
https://aprilhathcock.wordpress.com/2016/09/27/making-the-local-global-the-colonialism-of-scho larly-communication/ [Accessed August 21, 2017].

Hess, C. \& Ostrom, E., 2007. Introduction: An Overview of the Knowledge Commons. In C. Hess \& E. Ostrom, eds. Understanding knowledge as a commons. The MIT Press, pp. 4-26.

Kramer, B. et al., 2016. Defining the Scholarly Commons-Reimagining Research Communication. Report of Force11 SCWG Workshop, Madrid, Spain, February 25-27, 2016. Research Ideas and. https://doi.org/10.3897/rio.2.e9340.

Morris, S., 2005. The true costs of scholarly journal publishing. Learned publishing: journal of the Association of Learned and Professional Society Publishers, 18(2), pp.115-126. https://doi.org/10.1087/0953151053584975.

O'Donnell, D.P. et al., 2015. Aligning Open Access Publication with the Research and Teaching Missions of the Public University: The Case of the Lethbridge Journal Incubator (If "if"s and "and"s were pots and pans). The journal of electronic publishing: JEP, 18(3). http://doi.org/10.3998/3336451.0018.309.

O'Donnell, D.P., 2016a. Daniel Paul O'Donnell: But does it work in theory? Developing a generative theory for the scholarly commons. http://people.uleth.ca/ daniel.odonnell/Blog/but-does-it-work-in-theory-developing-a-generative-t heory-for-the-scholarly-commons [Accessed August 21, 2017].

O'Donnell, D.P., 2016b. Daniel Paul O'Donnell: But does it work in theory II. http://people.uleth.ca/ daniel.odonnell/Blog/but-does-it-work-in-theory-ii [Accessed August 21, 2017].

Ostrom, E., 1990. Governing the commons: The evolution of institutions for collective action, Cambridge, Cambridge University Press.

Piwowar, H. et al., 2017. The State of OA: A large-scale analysis of the prevalence and impact of Open Access articles, PeerJ Preprints. https://peerj.com/preprints/3119.

Research Information Network, 2008. Activities, costs and funding flows in the scholarly communications system in the UK, London: Research Information Network. http://www.rin.ac.uk/system/files/attachments/Activites-costs-flows-report.pdf [Accessed February 18, 2016].

Wikipedia contributors, 2017. Mertonian norms. Wikipedia, The Free Encyclopedia. https://en.wikipedia.org/w/index.php?title=Mertonian_norms\&oldid=782738713 [Accessed August 21, 2017]. 